\title{
Aspekte des Qualitätswesens in der ungarischen Hochschulbildung
}

\author{
Magdolna Nemeskéri
}

Die Erkenntnis, dass die Qualität in der Hochschulbildung eine wichtige Rolle spielt, hat im letzten Jahrzehnt zu einer wichtigen Änderung im Hochschulwesen gefuihrt. Nunmehr besteht die Einsicht, einerseits eine Qualitätskultur zu entwickeln und andererseits das Funktionsniveau der Hochschulen zu steigern. Hierbei ist anzumerken, dass das Qualitätswesen Gegenstand des Unterrichts sein kann, aber zugleich auch sein Mittel ist. Daher wurde oder wird in den Hochschulen ein eigenes zur Qualitätsauswertung anwendbares System geschaffen.

\section{Zusammenhang des Qualitätswesens und des Bildungswesens}

In Ungarn soll das Qualitätswesen - ähnlich den entwikkelten Ländern - das entscheidende Element der gesellschaftlich-wirtschaftlichen Entwicklung sein. Diese Erkenntnis ist in den vergangenen zehn Jahren allmählich in den Vordergrund gerüickt, und zwar wegen der in Ungarn generell vollzogenen Änderungen. Welche Änderungen waren das?

Nach der Änderung des politisches Systems in Ungarn wurde die Marktwirtschaft eingefuihrt, die außenwirtschaftlichen Beziehungen haben sich geändert. In den letzten Jahren hat sich die Wirtschaft stabilisiert, seit 1998 hat sich ihre Steigerung sogar beschleunigt, wozu die dynamische Entwicklung der Industrie deutlich beitrug. Die Entwicklung der ungarischen Wirtschaft ist von der Absicht, der Europäischen Union beizutreten, beeinflusst und bestimmt, was eine planmäßig geregelte Wirtschaftspolitik voraussetzt. Dazu gehört auch die Herausbildung einer neuen Qualitätsanschauung. Es ist ratsam, damit schon in der Schule zu beginnen.

Die veränderte Qualitätsanschauung ist eine direkte Folge der politischen und wirtschaftlichen Veränderungen. Sie wurde 1996 zusätzlich durch eine Regierungsverordnung verstärkt, die besagt, dass in der technischen Hochschulbildung auch Qualitätskenntnisse unterrichtet werden müssen. Das ist ein bedeutender Schritt im Prozess der Gestaltung einer neuen Qualitätsanschauung. Im nächsten Schritt muss der Unterricht des Qualitätswesens auch in die Humanbereiche einbezogen werden.

Die die Qualität und das System des Qualitätswesens begründende Qualitätswissenschaft ist keine technische und keine human-gesellschaftliche Wissenschaft, sondern eine die beiden Wissenschaftsbereiche in sich fassende gesellschaftlich-technische Wissenschaft.
Theorie und Praxis des Qualitätswesens basieren also auf humanen, gesellschaftlichen und technischen Kenntnissen. Folglich ist dessen Unterrichtung sowohl in der technischen als auch in der nichttechnischen Hochschulbildung möglich. Dies ist zur Ausgestaltung der Qualitätsanschauung auch nötig.

\section{Der Unterricht des Qualitätswesens}

Nach Untersuchungen in den Jahren 1993 und 1996 hat der Unterricht des Qualitätswesens ein sehr gemischtes Bild gezeigt. Dies trug dazu bei, dass auf den Unterricht des Qualitätswesens inzwischen großer Wert gelegt wird. Der Unterricht des Qualitätswesens kann zwei Ziele haben, das eine ist die bisher geschilderte Qualitätskultur, die Gestaltung der Qualitätsanschauung, das andere ist die Forderung vieler Wirtschaftsbereiche nach Experten, die auch eine entsprechende Ausbildung im Bereich des Qualitätswesens vorweisen können.

Mit Mitteln des PHARE-Programms wurde im Rahmen des TDQM-Programms unter Mitwirkung ausgezeichneter Autoren ein dreistufiges Lehrpaket erarbeitet (es enthält mehrere Fachbücher zum Qualitätswesen). Die einzelnen Teile des Lehrpakets haben je nach Anspruch der Nutzer unterschiedlichen Umfang.

Immer mehr Hochschulen unterrichten mittlerweile auch Qualitätswesen. Das ist in den oben genannten Stufen möglich: Es gibt Grundlagen zur Gestaltung der Anschauung und/oder man bildet entsprechend den Erwartungen der Industrie und anderer Wirtschaftsbereiche Fachleute im Bereich des Qualitätswesens aus. Zur Vielfältigkeit der Hochschullehre trägt bei, dass immer mehr Hochschulen auch außerhalb ihres klassischen Lehrsystems Fachleute fuir Qualitätswesen ausbilden.

Die Aufgaben des Unterrichts im Qualitätswesens sind immer noch vielfältig, was nicht bedeutet, dass die Ausgestaltung der Qualitätsanschauung oder die Ausbildung der Fachleute im Bereich Qualitätswesen nicht gut sind, sondern es wäre zweckmäßig, die Grundlagen in einigen Bereichen zu vereinheitlichen und die Ressourcen (Bewerbungen, Einbeziehung der Fachleute für Qualitätswesen, breitere Verwendung des schon zur Verfuigung stehenden Lehrstoffes etc.) noch intensiver zu nutzen.

In Ungarn beschäftigen sich mehrere Stellen mit dieser Frage. Die „Landeskonferenz der Lehrer für Qualitätswesen“ sowie die „Ungarische Qualitätswoche“, bei der ein ganzer Tag diesem Thema gewidmet wird, werden 
jährlich veranstaltet. In diesen Veranstaltungen geht es sowohl um den Unterricht des Qualitätswesens als auch um die Bewertung der Qualität des Unterrichts. Im Jahre 2000 erschien „Das Programm der Entwicklung des Unterrichts des Qualitätswesens“, das von einer Arbeitskommission der Ungarischen Qualitätsgesellschaft erarbeitet worden ist.

\section{Einige Gedanken aus dem Programm}

Die Gesellschaft nimmt die Qualitätskultur nur dann an und hält sie für wichtig, wenn alle Mitglieder und Gruppen sich über ihre Notwendigkeit im Klaren sind.

Die verschiedenen Gruppen sollen die Teile der Qualitätskultur kennen lernen, die für sie wichtig sind. Die Methoden und die Thematik des Unterrichts und der Bildung des Qualitätswesens für die nach Gesichtspunkten des Qualitätswesens unterschiedlichen Zielgruppen sollen aufeinander aufbauen und gut zueinander passen. Die Informationen über die Möglichkeiten sollen bekannt und zugänglich sein.

Der Unterricht und die Bildung des Qualitätswesens werden dann anerkannt, wenn sie nach bestimmten Anforderungen funktionieren.

Das heimische System für den Unterricht des Qualitätswesens soll den internationalen/europäischen Anforderungen entsprechen. Die Unterrichtssysteme, die über kein internationales/europäisches Anforderungssystem verfuigen, sollen ein einheitliches heimisches Anforderungsprofil erhalten.

Zur Verwirklichung des Unterrichts des Qualitätswesens sind entsprechende Rechtsvorschriften nötig.

Die Rechtsvorschriften fuir den Unterricht und die Bildung sollen gut überschaubar, ohne Überlappung und aufeinander abgestimmt sein.

Zur Verwirklichung des Unterrichts des Qualitätswesens ist eine finanzielles Umfeld nötig, das überschaubar ist, Kontinuität sichert und zur langfristigen Planung geeignet ist. Die Ausgestaltungs-, Instandhaltungs- und Entwicklungskosten des Unterrichts- und Bildungsprogramms des Qualitätswesens sowie die Finanzquellen hierfür sollen bekannt sein. Das Programm gibt Richtlinien vor, den Unterricht des Qualitätswesens in seinem System behan- deln zu können. Wenn eine Hochschule ihr Angebot um den Unterricht des Qualitätswesens erweitern will, muss sie sich mit den Fragen zu folgenden Komplexen beschäftigt haben (vgl. Abb. 1).

Welches Bildungsziel wird angestrebt? Wer wird der „Käufer“ der Dienstleistung sein?

Wenn diese Frage beantwortet ist, müssen die alle Informationen über die zu lehrenden Inhalte unter Einbeziehung der vorhandenen Forschungsergebnisse gesammelt und entsprechende Lehrpläne erstellt werden.

Welche Lehrinhalte sollen vermittelt werden?

Allgemeinbildende, die Qualitätsanschauung formierende Kenntnisse oder spezielle Kenntnisse, die geeignet sind, Fachleute fuir Qualitätswesen auszubilden.

Wie soll das System der zu lehrenden Kenntnisse sein? Damit aus dem System der zu lehrenden Kenntnisse ein Lehrstoff wird, müssen die Thematik, ein Aufeinanderaufbauen des Lehrstoffs sowie das Verhältnis zwischen Theorie und Praxis berücksichtigt werden.

In welcher Form soll unterrichtet werden? Im Direktstudium, im Fernstudium oder in Form des Fernunterrichts? Hierunter fallen die Aspekte Bildung, Lehren und Lernprozess.

\section{Die Interpretation des Qualitätswesens in der Hochschulbildung}

Wie erwähnt, berühren die wirtschaftlichen Veränderungen der vergangenen zehn Jahre das ungarische Hochschulwesen direkt und auch indirekt. Welche konkreten Änderungen sind hier zu verzeichnen?

\section{Expansion der Hochschulbildung}

Die Zahl der Studierenden an den ungarischen Hochschulen nimmt zu. Der große Zuwachs wirft organisatorische und personelle Probleme auf.

Entstehung neuer Hochschulbildungsinstitute

Die neu gegründeten Instituten bewegen sich auf unterschiedlichem Niveau. Daher müssen Bedingungen der Vergleichbarkeit geschaffen werden.

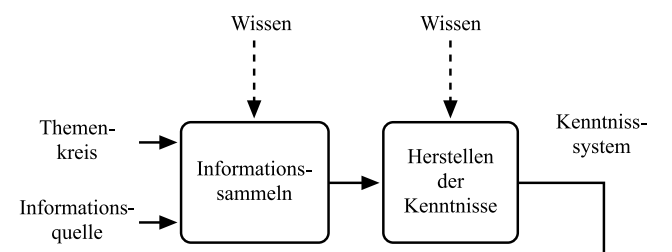

quelle
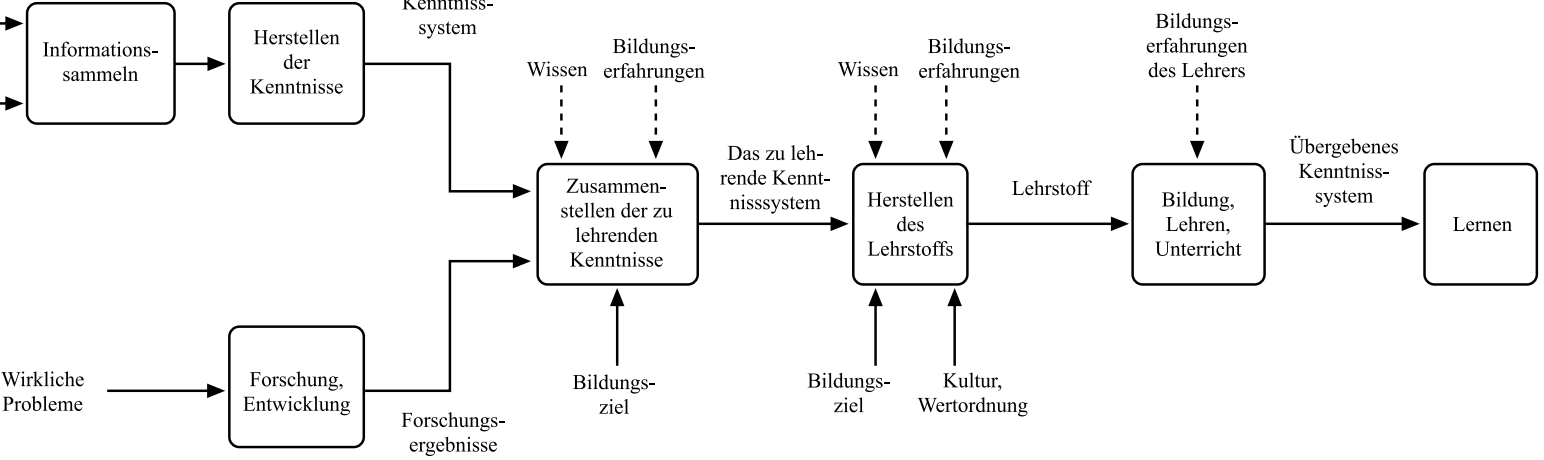

Abb. 1: Bildungsvorgang 


\section{Beitritt zur Europäischen Union}

Das stellt die ungarische Hochschulbildung vor ein doppeltes Problem. Man soll nicht nur beruicksichtigen, wie weit die Europäische Union jetzt ist, sondern wohin sie geht. Gleichzeitig entstehen neue Herausforderungen, die mit dem Beitritt zur Europäischen Union verbunden sind und denen sich die Europäische Union selbst stellen muss. Sowohl die ungarische Hochschulbildung als auch die Europäische Union sind mit vielerlei Änderungen der Jahrtausendwende konfrontiert, wie z. B.:

- Informatikgesellschaft: Die Grenzen von Lernen und Arbeit verwischen, die Rolle des Lehrers wird aufgeweicht.

- Globalisierungsvorgang: Der freie Fluss der Arbeitskräfte muss gesichert werden.

- Zivilisationsvorgang: Wegen der schnellen Veralterung der Kenntnisse gewinnen Qualifikation, Kreativität sowie die Fähigkeit zur Erneuerung und zur Weiterentwicklung neuer Kenntnisse an Bedeutung.

Die klassische Qualitätsanschauung der Hochschulbildung - Qualität als Begriff des Ausgezeichneten - wird immer mehr durch Aspekte, die für die Marktwirtschaft charakteristisch sind, abgelöst. Diese Situation fasst am Besten ein Vorschlag der Europäischen Union aus dem Jahr 1997 zusammen: „Die Qualität der wissenschaftlichen Forschung und die Qualität des Lehrstandes sind keine Garantie mehr für die Qualität des Unterrichts, denn genauso wichtig ist die Qualitätsauswertung der Organisation der Programme, der Lehrmethoden, des Institutsmanagements, der Struktur und der Kommunikation." Dieser Vorgang ist in Abbildung 2 dargestellt.

Die Qualitätsanschauung der Hochschulbildung bedeutet nur eine potenzielle Qualität, die effektive Qualität wird durch die Zufriedenheit der Käufer der Dienstleistungen bestimmt. Daraus folgt, dass es keinen Sinn hat, über die Qualität ohne ständige Rückkopplung der Käufermeinungen zu sprechen.

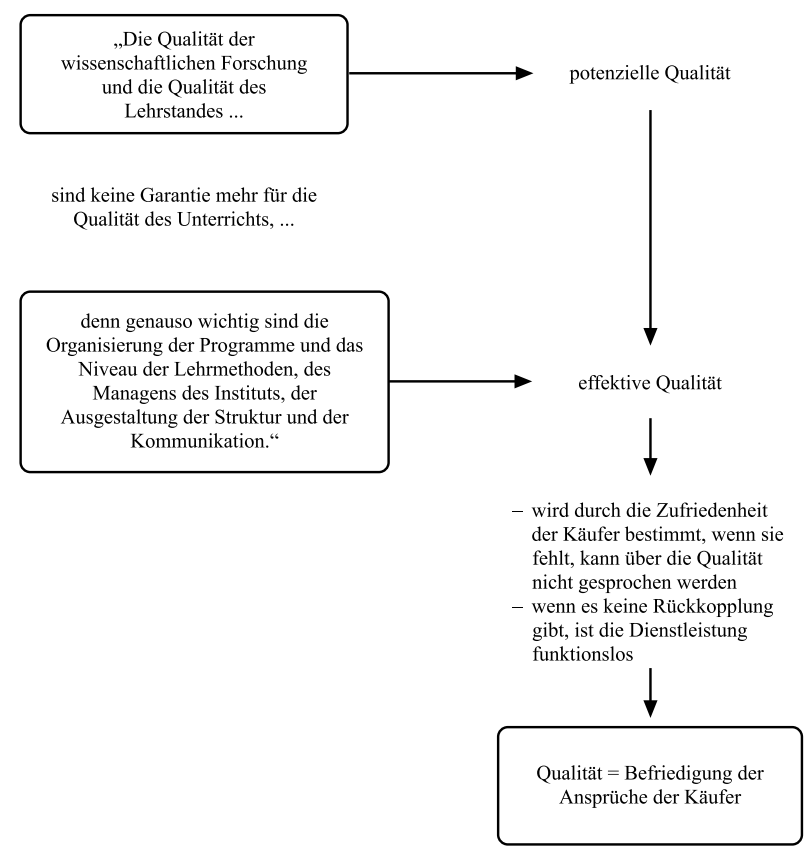

Abb. 2: Der Qualitätsbegriff der Europäischen Union für die Hochschulbildung
Unter anderem erfordern diese Aspekte, dass man sich mit dem Qualitätsmanagement der Hochschulen umfassend beschäftigt. Ungarn gehört zu denjenigen wenigen Ländern in Europa, die die Qualitätssicherung der Hochschulbildung durch Gesetze regeln. Das Hochschulbildungsgesetz hat die gesetzlichen Grundlagen fuir die Arbeit der Ungarischen Akkreditierungskommission (UAK) geschaffen, deren Aufgabe es ist, die Institute zu akkreditieren. Das ist der erste Schritt der Qualitätsauswertung der Hochschulbildung.

Die Akkreditierungskommission prüft,

- ob die Berufungserklärung, der Entwicklungsplan und die jetzige Lage des Instituts den Vorschriften des Gesetzes entsprechen,

- wie weit der Unterricht und die Forschungsarbeit des Instituts dem in der Berufungserklärung Beschriebenen entsprechen,

- wie zeitgemäß die Berufslehrpläne des Instituts sind,

- ob die Fachkenntnisse der Lehrer dem niveauvollen Unterricht der zeitgemäßen Lehrpläne entsprechen,

- ob die Infrastruktur des Instituts, sein inneres System und seine Außenbeziehungen bei der Unterrichtsund Forschungstätigkeit entsprechend helfen.

Die UAK äußert ihre Meinung über

- die Gründung und Anerkennung einer Universität bzw. Hochschule,

- den Beginn und die Einstellung eines Faches,

- die Anforderungen der Bildung,

- den Doktoren- und Habilitationsstatut einer Universität.

Eine Frage ist, wie weit die im Hochschulbildungsgesetz vorgeschriebene Akkreditierung eines Instituts die Qualität sichert. Da der Akkreditierungsvorgang prüft, ob der Personalbestand und die sachlichen Bedingungen dem Unterricht im Institut entsprechen, und auf viele andere Bereiche der Hochschulbildung nicht eingeht, hat die Regierung ihrerseits die Erwartung formuliert - mit Rücksicht auf die heimischen und ausländischen Umstände -, dass die Hochschuleinrichtungen im Interesse der Durchschaubarkeit und der Vergleichbarkeit ein Qualitätsmanagementsystem ausbauen, dessen Form die autonomen Institute selbst auswählen können.

Es gibt mehrere Möglichkeiten, ein Qualitätsmanagementsystem aufzubauen. Ein langfristiges Ziel ist, ein umfassendes Qualitätsmanagement zu etablieren. Eine Zwischenstation dessen kann ein Qualitätsmanagementsystem sein, das nach dem in Ungarn im Jahre 2001 in Kraft getretenen Standard ISO 9004:2000 entwickelt wird. Die Zeitgemäßheit des neuen Standards betonen die acht Grundprinzipien, die größtenteils mit den Grundprinzipien des umfassenden Qualitätsmanagements, auch Total Quality Management (TQM) genannt, übereinstimmen.

Die acht Grundprinzipien sind:

1. Kundenzentrierung: Die Organisationen hängen von ihren Kunden ab, deshalb müssen sie die gegenwärtigen und die zukünftigen Bedürfnisse der Kunden 
kennen, die Anforderungen der Kunden befriedigen und sich bemühen, die Erwartungen der Kunden zu uibertreffen.

2. Leitung: Die Leiter kümmern sich um die Einhaltung der Ziele und die Leitung der Organisation. Sie bringen eine innere Umgebung zustande, in der die Mitarbeiter am Erreichen der Organisationsziele in vollem Maße teilnehmen.

3. Beteiligung der Mitarbeiter: Das Wesen der Organisation sind auf jeder Stufe die Mitarbeiter und ihre vollständige Beteiligung macht es möglich, ihre Fähigkeiten zugunsten der Organisation zu nutzen.

4. Annäherung von der Seite Prozessanschauung: Das gewünschte Ergebnis kann effektiver erreicht werden, wenn die Tätigkeiten und die Ressourcen als Prozess gesteuert werden.

5. Systemanschauung in der Leitung: Die Identifizierung, das Verstehen und die Leitung der miteinander zusammenhängenden Prozesse tragen dazu bei, dass die Organisation ihre Ziele erfolgreich und effektiv erreicht.

6. Ständige Entwicklung: Ständiges Ziel der Organisation soll es sein, die Tätigkeit der Organisation kontinuierlich umfassend weiterzuentwickeln.

7. Entscheidungsfindung nach Fakten: Die erfolgreiche Entscheidungsfindung basiert auf der Analyse der Angaben.

8. Gegenseitig vorteilhafte Kontakte mit Kooperationspartnern: Das steigert die wertschaffende Tätigkeit von beiden.

Von den acht Grundprinzipien möchte ich drei hervorheben, die beim Ausbau des Qualitätsmanagementsystems besonders wichtig sind. Eines ist das Prinzip der Annäherung von der Seite der Prozessanschauung. Seine Bedeutung besteht darin, dass die Hochschule ihre wichtigen Prozesse bestimmt. Sie definiert diese eindeutig, legt die Eingänge, Ausgänge und Charakterzuige des Prozesses fest und bestimmt die Verknuipfungen zwischen den verschiedenen Prozessen. Ein wichtiger Prozess innerhalb einer Hochschule ist die Lehre, aber auch die Entwicklung der Studiengänge und die Studienberatung, die diesen Prozess bedienen.

Ein weiteres Prinzip ist die Kundenzentrierung. Die Hochschule muss bestimmen, wen sie für ihre Kunden hält. Einer der wichtigsten Kunden ist der Studierende, aber Kunden sind auch der Arbeitgeber, der Hochschulabsolventen einstellt, die Mittelschule, die der Hochschule den Studienanfänger „liefert“, und natürlich alle Mitarbeiter und Lehrer, die an der Hochschule arbeiten. Um die Ansprïche ihrer Kunden befriedigen zu können, muss die Hochschule diese erst einmal kennen. Sie muss darüber hinaus ständig darauf achten, ob und wie die Kunden mit den angebotenen Dienstleistungen zufrieden sind. So ist die Zufriedenheit der Studierenden zu ermitteln im Hinblick auf z. B. den Bildungsprozess, den Aufbau und die Zeitgemäßheit des Lehrstoffs, die sachlichen und technischen Bedingungen des Unterrichts usw. Die Zufriedenheit der Hochschulmitarbeiter sollte gemessen werden an $\mathrm{z}$. B. ihrer Zufriedenheit mit den Karrieremöglichkeiten, mit dem Arbeitsklima usw. Das Hochschule muss also ihren Kundenkreis exakt bestimmen und dessen Bedürfnisse und Zufriedenheit während der eigenen Verbesserung fortwährend beachtet.

Daran schließt sich das dritte wichtige Grundprinzip an, die ständige Entwicklung. Sie kann verwirklicht werden, wenn die Hochschule die Ergebnisse der Messung der Kundenzufriedenheit als Grundlage für ihre Verbesserung nutzt. Werden die Messergebnisse zur richtigen Zeit und an der richtigen Stelle verwendet, funktioniert auch der Regelungskreis, durch den man in den Prozess eingreifen und den Prozess so gut wie möglich gestalten kann. Wenn die Hochschule diese Prinzipien bei der Einrichtung ihres Qualitätsverbesserungsprogramms berücksichtigt, bekommt sie ein gut funktionierendes, überschaubares Qualitätsmanagementsystem.

Um die Funktionsfähigkeit des Qualitätsmanagementsystems in der Hochschulbildung herzustellen, bietet sich als weitere Möglichkeit die Anwendung des „Business Excellence“-Modells der EFQM (European Foundation for Quality Management) an. Es wird seit 1996 auch in Ungarn in verschiedenen Wirtschaftsbereichen verwendet. Abbildung 3 zeigt die Adaption des EFQMModells für den Unterricht. Die Qualitätsauswertung nach diesem Modell ist auch für den Vergleich der Hochschulen geeignet.

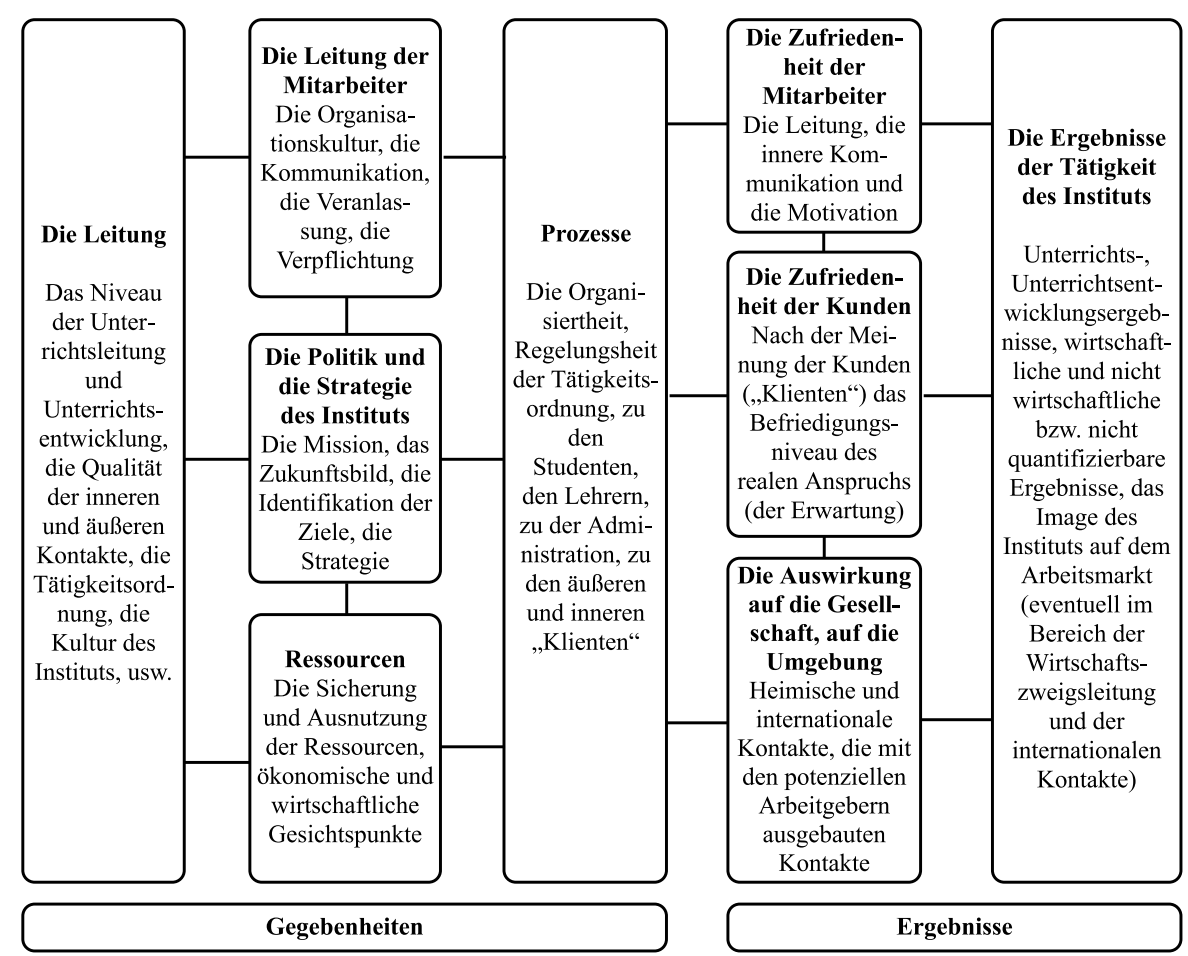

Abb. 3: Die Verwendung des EFQM-Modells für den Unterricht 


\section{Literatur}

Veress, Gábor; Kovács. Károly: Der Unterricht des Qualitätswesens in der nicht technischen Hochschulbildung. Herausgabe Der neunten ungarischen Veress, Gábor Woche, Seiten: 10-12.

Veress, Gábor: Das Qualitätsmanagement der Hochschulbildung. Technischer Verlag, Budapest 2000

Dinya, László: Die Qualitätssicherung der Hochschulbildung und der Anschluss der EU. Ungarische Hochschulbildung 1999/4. Seiten: 30-31.

\section{Autor}

\section{Magdolna Nemeskéri}

Technische Hochschule Budapest

Kandó Kálmán Fakultät für Elektroingenieurwesen

Institut für Computertechnik

Budai út 45, 8000 Székesfehérvár, Ungarn

E-Mail: nemeskeri@szgti.bmf.hu 